\title{
Neferine induces apoptosis by modulating the ROS-mediated JNK pathway in esophageal squamous cell carcinoma
}

\author{
KANG AN", YUEHAN ZHANG*, YINGJIAO LIU, SHENGXI YAN, ZHAOWEI HOU, MENG CAO, \\ GUANGKUO LIU, CONGCONG DONG, JUNCHA GAO and GAIFANG LIU
}

Department of Gastroenterology, Hebei General Hospital, Shijiazhuang, Hebei 050051, P.R. China

Received February 27, 2020; Accepted June 2, 2020

DOI: $10.3892 /$ or.2020.7675

\begin{abstract}
Current treatments for esophageal squamous cell carcinoma (ESCC) have limited efficacy. Therefore, the development of novel therapeutic targets to effectively manage the disease and boost survival rates is imperative Neferine, a natural product extracted from Nelumbo nucifera (lotus) leaves, has been revealed to inhibit the growth of hepatocarcinoma, breast cancer and lung cancer cells. However, its effect on ESCC is unknown. In the present study, it was revealed that neferine exerted anti-proliferative effects in ESCC. It was also revealed that it triggered arrest of the $\mathrm{G}_{2} / \mathrm{M}$ phase and enhanced apoptosis of ESCC cell lines. Moreover, its ability to trigger accumulation of reactive oxygen species (ROS) and activate the c-Jun N-terminal kinase (JNK) pathway was demonstrated. Further study revealed how $\mathrm{N}$-acetyl cysteine (NAC), a ROS inhibitor, attenuated these effects, demonstrating that ROS and JNK inhibitors mediated a marked reversal of neferine-triggered cell cycle arrest and apoptosis in ESCC cells. Finally, it was revealed that neferine was involved in the inhibition of Nrf2, an antioxidant factor. Collectively, these findings demonstrated the antitumor effect of neferine in ESCC, through the ROS-mediated JNK pathway and inhibition of $\mathrm{Nrf2}$, indicating its potential as a target for development of novel and effective therapeutic agents against ESCC.
\end{abstract}

\section{Introduction}

Esophageal carcinoma (EC), that occurs in the squamous or glandular epithelium of the esophagus, represents the predominant type of gastrointestinal tumors $(1,2)$. EC is

Correspondence to: Dr Gaifang Liu, Department of Gastroenterology, Hebei General Hospital, 348 West HePing Boulevard, Shijiazhuang, Hebei 050051, P.R. China

E-mail:srmxhk@163.com

*Contributed equally

Key words: Neferine, esophageal squamous cell carcinoma, apoptosis, reactive oxygen species, c-Jun $\mathrm{N}$-terminal kinase pathway further divided into esophageal squamous cell carcinoma (ESCC) and esophageal adenocarcinoma (3). Approximately 300,000 esophageal carcinoma-related deaths occur every year worldwide, with its incidence and mortality rates varying widely across countries. In China, studies indicate that $\sim 150,000$ people succumb to EC every year, with $>90 \%$ of them diagnosed with ESCC (4). Although some progress has been achieved with regard to diagnosis and treatment of ESCC, its five-year survival rate remains low (2). It is, therefore, imperative to develop antitumor drugs that effectively improve the overall survival rates, while conferring low toxicity to patients. During the development of antitumor drugs, numerous focus has been directed towards active compounds from natural products. For example, paclitaxel a new anti-microvascular drug extracted from the bark of Taxus brevifolia, has been used in the treatment of ESCC and ovarian carcinoma $(5,6)$. Similarly, vincristine, an alkaloid extracted from Catharanthus roseus, is widely applied in the treatment of germinoma (7) and lymphoma (8). In addition, Neferine (Fig. 1A), an alkaloid extracted from Nelumbo nucifera (lotus), has been revealed to actively reduce blood lipid levels and anti-inflammation, and recently it was reported to exert antitumor effects in multiple tumor cells $(9,10)$. Xu et al (11) revealed that neferine suppressed the proliferation of ovarian carcinoma cells by provoking autophagy. In osteosarcoma, neferine inhibited cell proliferation by triggering cell cycle arrest (12). However, its effects on ESCC remain unknown.

Abnormal alterations in the cell cycle is a hallmark of cancer, and has been extensively exploited as a major target for development of treatment therapies $(13,14)$. Previous studies have demonstrated that cyclins, along with cyclin-dependent kinases (CDKs), are key regulators of cell cycle progression. For instance, cyclin B1 is required to initiate mitosis by modulating phosphorylation or dephosphorylation of proteins (15). In addition, a series of CDKs, such as p21, have been reported to function as regulators during activation of cyclin B1 (16).

Apoptosis refers to the active and orderly death of cells that maintains homeostasis of the internal environment under physiological or pathological conditions $(17,18)$. Exposure of cells to internal pro-apoptotic factors, such as activators of oncogenes, agents that cause DNA damage, cell hypoxia, and deficiency of cell growth factors, has been revealed to activate apoptosis of mitochondrial cells (19). Previous studies have 
demonstrated that dysregulation of apoptosis can result in a variety of human diseases, including development and regression of tumors $(20,21)$. In fact, dysregulation-related resistance to apoptosis is one of the causes for tumorigenesis (22).

Reactive oxygen species (ROS) has been implicated in tumorigenesis. Particularly, a moderate ROS level is required for cell proliferation but excessive production of ROS induces apoptosis causing cell death (23). Furthermore, ROS has been revealed to modulate various pathways, including the c-Jun N-terminal kinase (JNK), which is a key regulator of apoptosis $(24,25)$. Recent studies demonstrated that the level of ROS could be tightly controlled by the cellular antioxidant system. The transcription factor nuclear factor erythroid 2-related factor 2 ( Nrf2) is deemed as the master regulator of the antioxidant system. Although consistently exposed to a high level of ROS, cancer cells could survive by upregulating the expression of Nrf2. In cerebral ischaemia/reperfusion injury, the oxidative damage caused by overproduced ROS could be removed by enhancing the expression of Nrf2 (26-28). Hence, Nrf2 is usually regarded as an upstream molecule of ROS.

The present study investigated the potential anticancer activity of neferine in ESCC cells. It was revealed that neferine induced cell cycle arrest and apoptosis in these cells. Mechanistically, neferine induced ROS-mediated activation of the JNK signaling pathway by inhibiting Nrf2. Collectively, these findings demonstrated that neferine holds great promise as a treatment for ESCC.

\section{Materials and methods}

Reagents. Neferine (purity $>98 \%$ ) was acquired from Selleckchem, whereas tert-Butylhydroquinone (tBHQ; B105351), N-acetyl-L-cysteine (NAC; A105421) and SP600125 (SP; S125267) were purchased from Shanghai Aladdin Biological Technology Co., Ltd. Neferine was dissolved in DMSO (Sigma-Aldrich; Merck KGaA) at a concentration of $100 \mathrm{mM}$ as a primary stock solution and the desired concentration of neferine for each experiment was obtained via thinned with RPMI-1640 medium with $10 \%$ FBS, immediately before use. The concentration of DMSO was lower than 1:3,000 in all experiments.

Cell lines and cultures. Cancer cell lines, KYSE30, KYSE150 and KYSE510, were obtained from Fengh Biotech and maintained in RPMI-1640 medium, supplemented with $10 \%$ FBS (HyClone; GE Healthcare Life Sciences). The cultures were incubated at $37^{\circ} \mathrm{C}$, and $5 \% \mathrm{CO}_{2}$.

Cell viability assay. Approximately 4,000 cells/well, were seeded overnight in 96-well plates, then treated with varying concentrations $(0,5,10,15,20$ and $30 \mu \mathrm{m})$ of neferine for 24 and $48 \mathrm{~h}$ at room temperature. Cell viability was determined by detecting the absorbance at OD $450 \mathrm{~nm}$ using $10 \mu \mathrm{l}$ Cell Counting Kit-8 (NCM Biotech) according to the manufacturer's instructions.

Clone formation assay. Approximately 1,000 cells/well, earlier incubated with the aforementioned neferine concentrations, were seeded in 6-well plates and cultured for 14 days. The colonies were then washed with PBS, fixed with $75 \%$ ethanol at room temperature for $15 \mathrm{~min}$ and stained using $0.1 \%$ crystal violet for another $15 \mathrm{~min}$. Finally, images were obtained using a digital camera (Olympus) and the number of forming colonies ( $>10$ cells per colony) was measured by Gel Imaging Analysis System (Syngene).

Apoptosis assays. Approximately $5 \times 10^{4}$ cells were collected, following overnight incubation with various neferine concentrations $(0,10,15$ and $20 \mu \mathrm{m})$, then incubated with $5 \mu \mathrm{l}$ Annexin V-FITC and $5 \mu 1$ propidium iodide (Neobioscience) in darkness for $15 \mathrm{~min}$ at room temperature. Thereafter, apoptosis was assessed using a flow cytometer (BD Biosciences) and the results were analyzed with FlowJo analysis software (version 10.0; FlowJo LLC).

Analysis of the cell cycle. Approximately $2 \times 10^{5}$ cells were plated in a well of 6-well plates for $24 \mathrm{~h}$, followed by another $24 \mathrm{~h}$ incubation with neferine at $37^{\circ} \mathrm{C}$. The cultures were then collected and fixed with ice-cold $70 \%$ ethanol at $4^{\circ} \mathrm{C}$ overnight, followed by determination of cell cycle distribution using a Cell Cycle Detection Kit (Nanjing KeyGen Biotech Co., Ltd.) according to the manufacturer's instructions.

Western blot analysis. Profiles of protein expression were analyzed using western blot as previously described (21). The primary antibodies used in the present study included E-cadherin (product code ab194982), N-cadherin (product code ab18203), cyclin B1 (product code ab32053), p21 (product code ab109520), Bax (product code ab32503), Nrf2 (product code ab137550), and phosphorylated (p)-JNK (product code ab4821) (all from Abcam), caspase-3 (cat. no. 19677-1-AP), Bcl-2 (cat. no. 12789-1-Ap), Beclin-1 (cat. no. 11306-1-AP), JNK (cat. no. 24164-1-AP) and caspase-9 (cat. no. 10380-1-AP) (all from ProteinTech Group, Inc.). $\beta$-actin (cat. no. A19788) (Sigma-Aldrich; Merck KGaA) was included as an internal control. The blots were then incubated with horseradish peroxidase (HRP)-conjugated secondary antibodies including anti-mouse IgG (dilution 1:2,000; cat no. 7076) and anti-rabbit (dilution 1:2,000; cat. no. 7074; both from Cell Signaling Technology, Inc.). The protein bands were analyzed using ImageQuant LAS 4000/4010 (GE Healthcare).

Determination of ROS production. Levels of ROS produced were measured using a ROS Assay kit (Nanjing KeyGen Biotech Co., Ltd.), according to the manufacturer's instructions, then the results analyzed using fluorescent microscopy (magnification, x100) and a flow cytometer (FACScan; BD Biosciences) with FlowJo analysis software (version 10.0; FlowJo LLC).

Statistical analyses. All experiments were conducted using at least three replicates, and the results were presented as the means \pm standard deviations (SD) of the means. A one-way analysis of variance (ANOVA) was used to compare differences among multiple groups with Tukey's post hoc test. An unpaired Student's t-test was employed for comparisons between two groups. All data analyses were performed in GraphPad Prism version 7 (GraphPad Software, Inc.) or SPSS 22.0 (IBM Corp). 
A

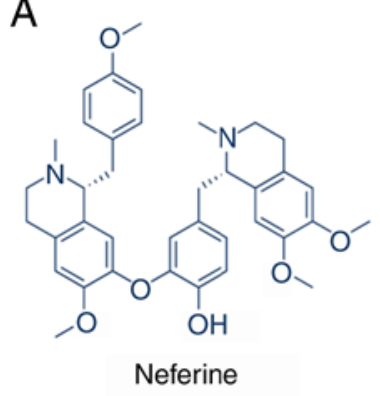

B

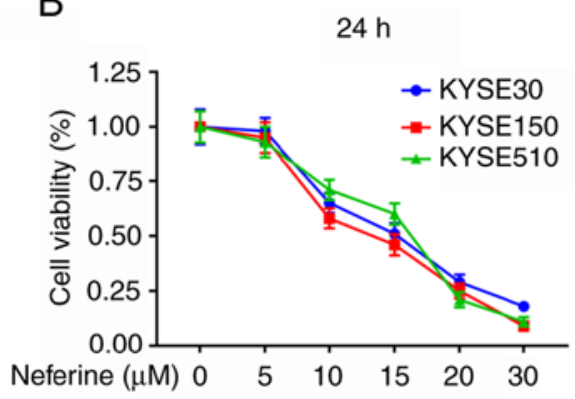

C

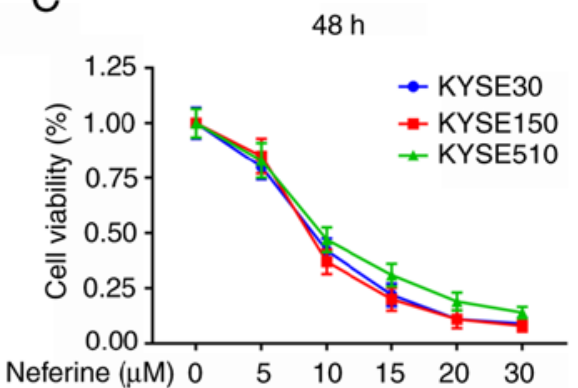

D $\quad$ Neferine $(\mu \mathrm{M})$

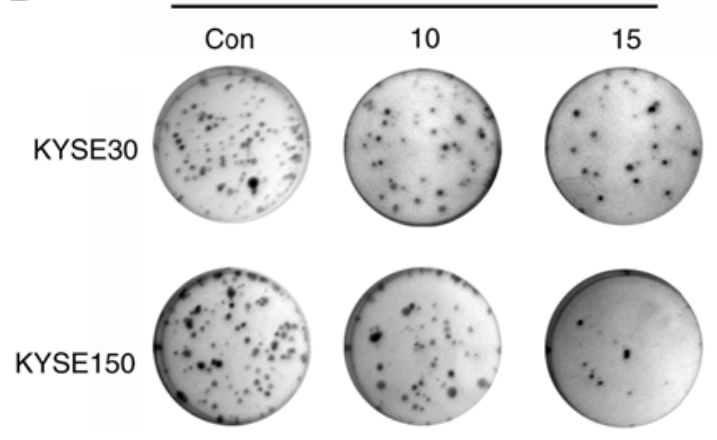

E

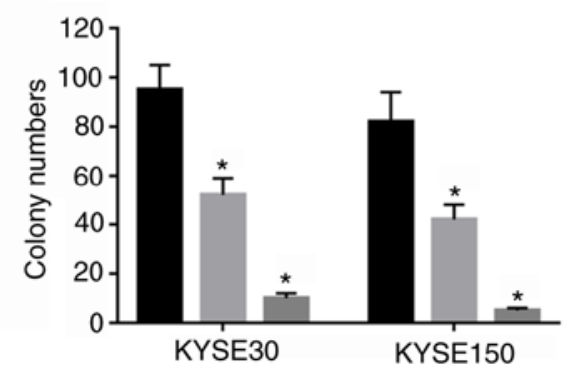

- Control

$10 \mu \mathrm{M}$

$15 \mu \mathrm{M}$

Figure 1. Anti-proliferative effects of neferine in ESCC cell lines. (A) Neferine structure. (B and C) KYSE30, KYSE150 and KYSE510 cells were treated with neferine $(0,10$ and $15 \mu \mathrm{M})$ for (B) $24 \mathrm{~h}$ and (C) $48 \mathrm{~h}$. Cell viability was detected by the CCK-8 assay. (D and E) Results of colony formation assays in KYSE30 and KYSE150 cells incubated with neferine $(0,10$ and $15 \mu \mathrm{M}){ }^{*} \mathrm{P}<0.05$ vs. $0 \mu \mathrm{M}$ of neferine or vs. the control. ESCC, esophageal squamous cell carcinoma; CCK-8, Cell Counting Kit-8.

\section{Results}

Neferine suppresses cell proliferation. To explore the antiproliferative activity of neferine on ESCC cells, three cell lines (KYSE30, KYSE150 and KYSE510) were incubated with varying $(0,5,10,15,20$ or $30 \mu \mathrm{M})$ concentrations for $24 \mathrm{~h}$ (Fig. 1B) and $48 \mathrm{~h}$ (Fig. 1C) and the viability of the cells was determined using a CCK- 8 assay. The results revealed that neferine hindered the proliferation of the three ESCC cell lines. Notably, the $\mathrm{IC}_{50}$ values, after $24 \mathrm{~h}$, were $14.16 \pm 0.911$, $13.03 \pm 1.162$, and $14.67 \pm 1.353 \mu \mathrm{M}$ in KYSE30, KYSE150, and KYSE510 cells, respectively. A colony formation assay, performed to evaluate the long-term effects of neferine on proliferation of ESCC cells (Fig. 1D) revealed suppression of clonogenicity in KYSE30 and KYSE150 relative to the control group (Fig. 1E). Collectively, these results indicated that neferine hindered proliferation of ESCC cells.

Neferine induces cell cycle arrest. To ascertain whether neferine hinders ESCC proliferation by regulating cell cycle distribution, KYSE30 and KYSE150 cells were exposed to 4 different neferine concentrations $(0,10,15$ and $20 \mu \mathrm{M})$ for $24 \mathrm{~h}$, and then cell cycle distribution was analyzed using flow cytometry (Fig. 2A). A higher $\mathrm{G}_{2} / \mathrm{M}$-phase distribution in neferine-treated KYSE30 and KYSE150 cells relative to the control group (Fig. 2B) was revealed. The proportion of $\mathrm{G}_{2} / \mathrm{M}$ phase cells in KYSE30 cells was $9.7 \pm 0.91 \%$ in the untreated group, while the percentages from neferine treatment were $15.1 \pm 1.37(10 \mu \mathrm{M}), 22.8 \pm 1.84 \%(15 \mu \mathrm{M})$ and $33.0 \pm 3.25 \%(20 \mu \mathrm{M})$. In KYSE150 cells, the proportions of $\mathrm{G}_{2} / \mathrm{M}$ phase cells were of $10.2 \pm 0.92 \%$ (untreated), $16.3 \pm 2.32 \%$
$(10 \mu \mathrm{M}), 25.3 \pm 2.45 \%(15 \mu \mathrm{M})$ and $33.2 \pm 3.41 \%(20 \mu \mathrm{M})$. Next, western blot analysis was used to detect the expression of cyclin B1 and p21, and the results revealed that neferine mediated a downregulation of cyclin B1, but upregulation of p21 expression (Fig. 2C and D). These results indicated that neferine inhibited growth of ESCC cells by modulating cell cycle-related proteins.

Neferine induces cell apoptosis. Apoptosis assays were performed to investigate the ability of neferine to inhibit proliferation of ESCC. The results revealed significantly higher apoptosis in neferine-treated KYSE30 and KYSE150 cells, relative to the control group (Fig. 3A and B). Specifically, apoptosis rates in KYSE30 cells were $2.13 \pm 0.67 \%$ for the untreated group, while those in neferine-treated cells were $16.55 \pm 2.45 \%$ $(10 \mu \mathrm{M}), 24.2 \pm 3.67 \%(15 \mu \mathrm{M})$ and $53.2 \pm 5.31 \%(20 \mu \mathrm{M})$. In KYSE150 cells, the apoptotic rates were $3.21 \pm 0.72 \%$ (untreated), $15.31 \pm 3.22 \%(10 \mu \mathrm{M}), 27.3 \pm 3.45 \%(15 \mu \mathrm{M})$ and $43.2 \pm 4.21 \%(20 \mu \mathrm{M})$. Furthermore, neferine treatment significantly increased the expression levels of cleaved PARP as well as cleaved caspase-3 and 9, but downregulated the expression of Bcl-2 (Fig. 3C and D). These findings indicated that neferine successfully induced apoptosis in ESCC cells.

Neferine increases ROS production and activates the JNK pathway. Neferine has been demonstrated to cause excessive ROS production leading to apoptosis (12). Based on this, its ability to induce ROS production in ESCC cells was explored by treating KYSE30 and KYSE150 cells with 10, 15 and $20 \mu \mathrm{M}$ of neferine for $12 \mathrm{~h}$ (Fig. 4A), and then sequentially exposing them to $15 \mu \mathrm{M}$ for 6,12 and $24 \mathrm{~h}$ (Fig. 4B). The 
A

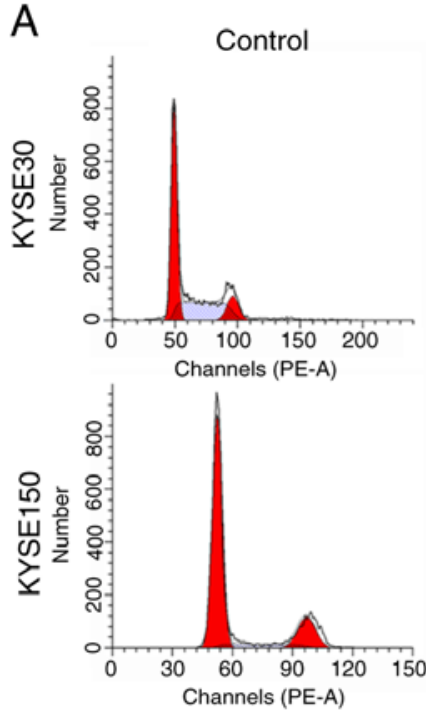

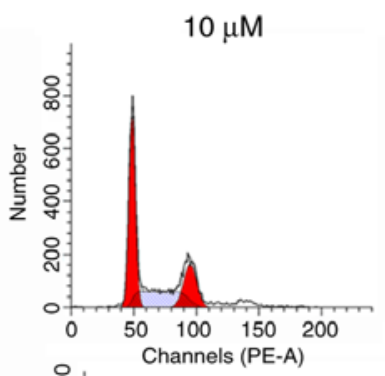

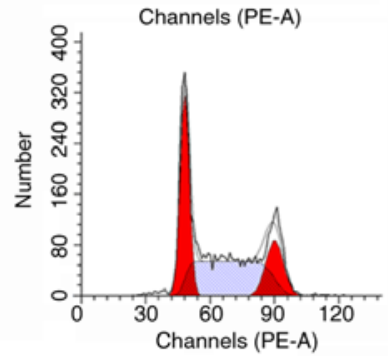

G2

$\mathrm{S}$
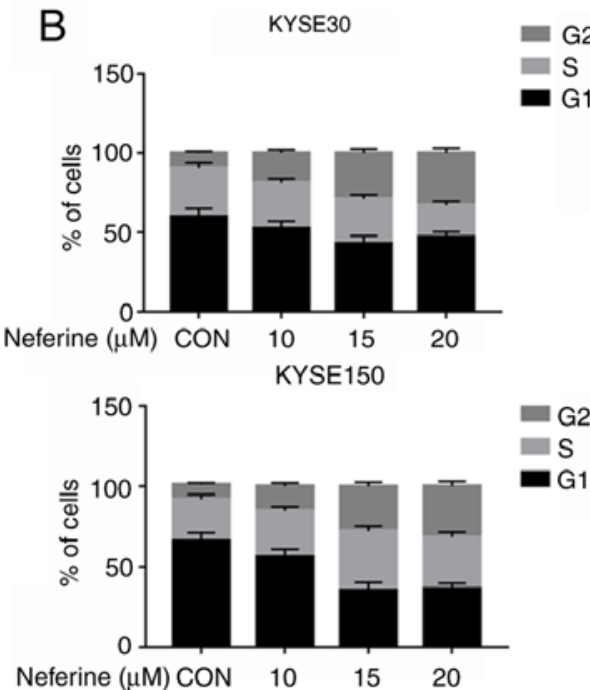

Neferine $(\mu \mathrm{M}) \mathrm{CON}$

1520
D

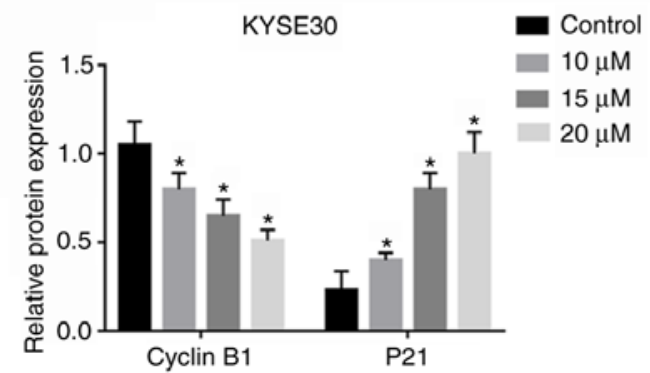

C
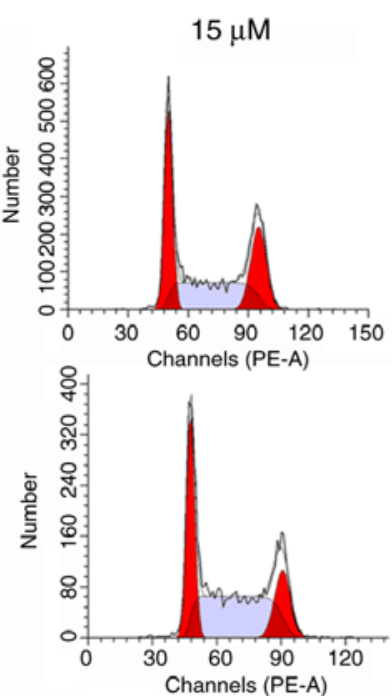

$20 \mu \mathrm{M}$
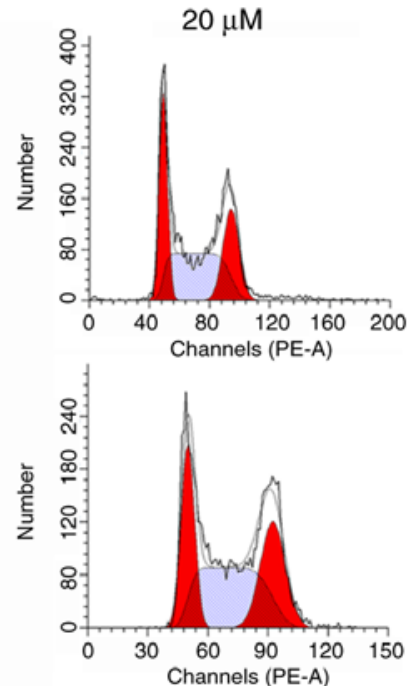
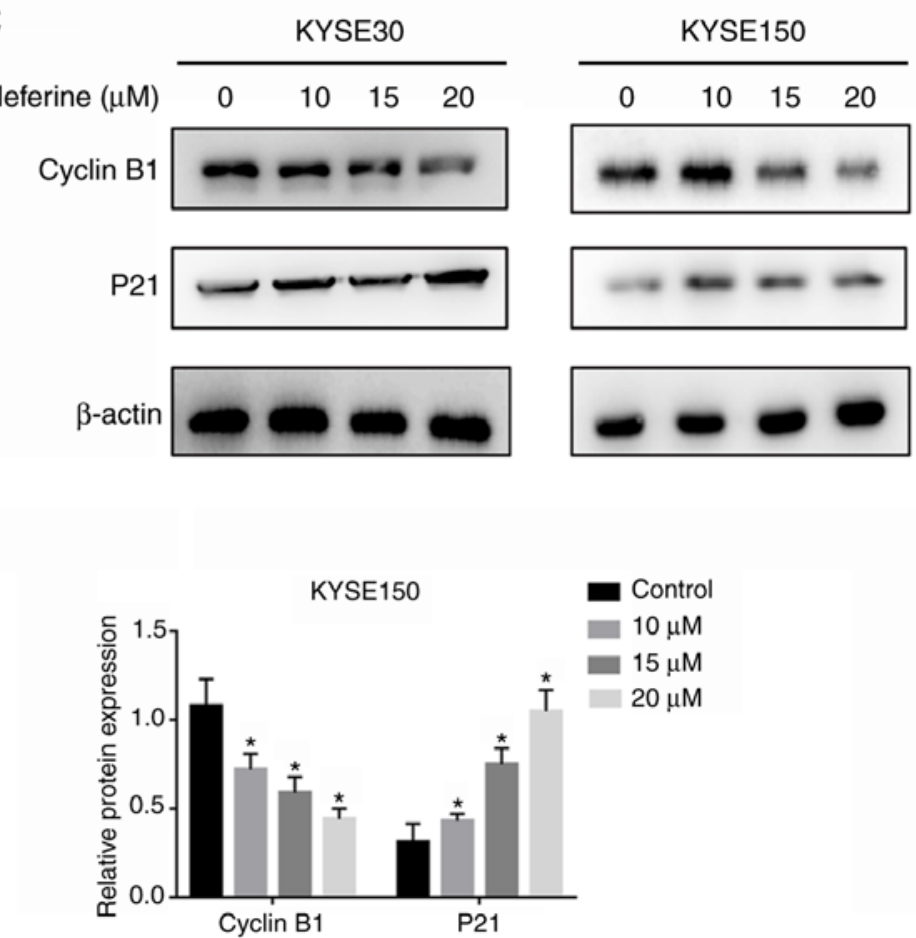

Figure 2. Neferine-triggered cell cycle arrest in ESCC cells. (A and B) KYSE30 and KYSE150 cells were incubated with neferine (0, 10 , 15 and $20 \mu \mathrm{M})$ for $24 \mathrm{~h}$, and cell cycle distribution was assessed via flow cytometry. (C and D) KYSE30 and KYSE150 were incubated with neferine ( 0 , 10,15 and $20 \mu \mathrm{M})$ for $24 \mathrm{~h}$. The expression of cyclin B1 and p21 was detected via western blot analysis. ${ }^{*} \mathrm{P}<0.05$ vs. the control. ESCC, esophageal squamous cell carcinoma.

results revealed significantly higher intracellular ROS levels at 6,12 and $24 \mathrm{~h}$, in neferine-treated cells relative to the control group. Similarly, higher ROS levels were detected in KYSE30 and KYSE150 cells exposed to higher, compared to lower neferine concentrations (Fig. 4A). Then, the antioxidant, NAC was analyzed, using fluorescence microscopy (Fig. 4C) and flow cytometry (Fig. 4D) and it was revealed that this ROS inhibitor significantly reduced the production of ROS in ESCC cells following neferine treatment. Numerous studies have reported a close relationship between ROS production and activation of the JNK pathway $(29,30)$. Therefore, the expression of JNK pathway-related proteins was assessed using western blot analysis and the results revealed significantly higher levels of JNK phosphorylation in neferine-treated ESCC cells (Fig. 4D and E).

Neferine triggers cell cycle arrest and apoptosis by increasing $R O S$ production. Since the antioxidant NAC could significantly reduce the generation of ROS, subsequently, the effects of neferine-mediated ROS accumulation on apoptosis and cycle 

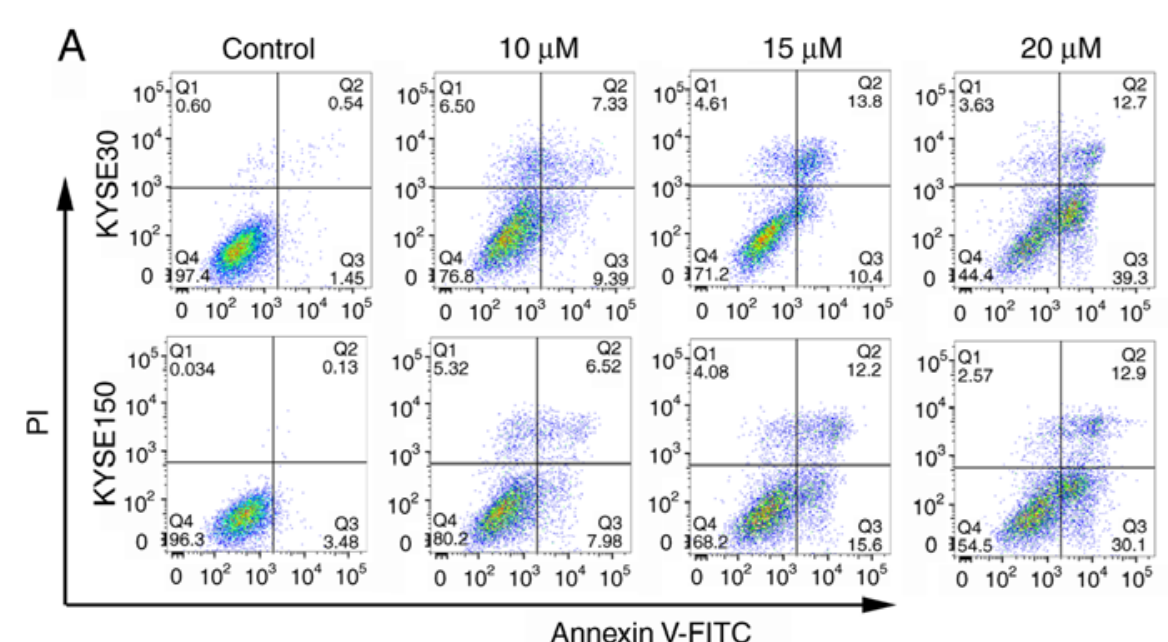

B

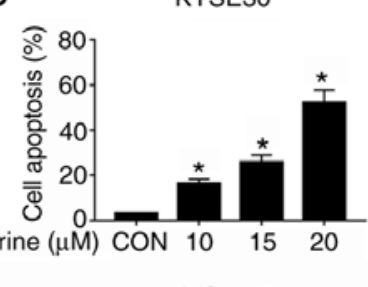

Neferine $(\mu \mathrm{M}) \mathrm{CON} 10 \quad 15$
KYSE150

C

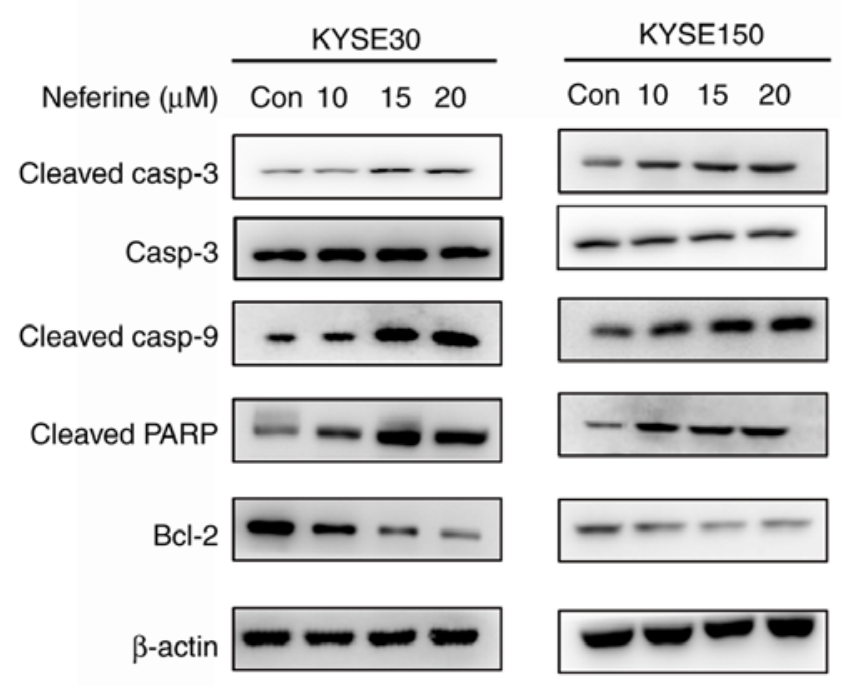

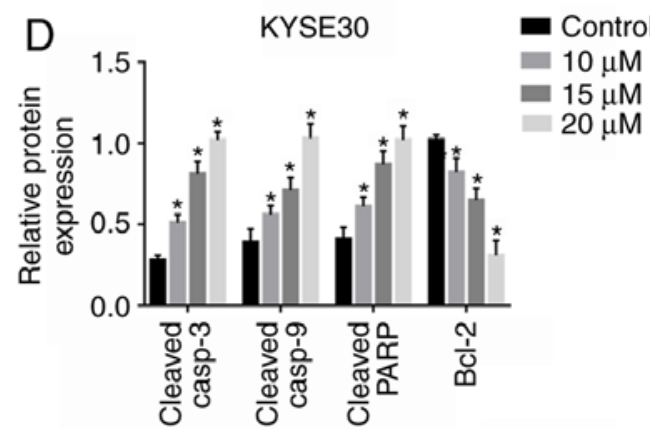

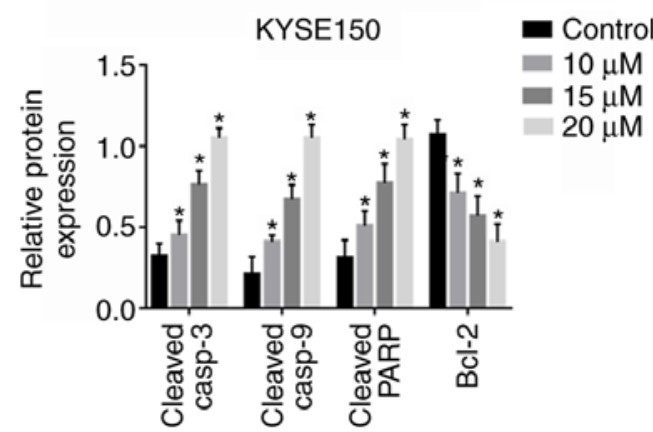

Figure 3. Neferine induces apoptosis in ESCC cells. (A and B) KYSE30 and KYSE150 cells were incubated with neferine (0, 10,15 and $20 \mu \mathrm{M})$ for $24 \mathrm{~h}$, and cells apoptosis was determined via flow cytometry. (C and D) KYSE30 and KYSE150 were incubated with neferine $(0,10,15$ and $20 \mu \mathrm{M})$ for $24 \mathrm{~h}$, after which the expression of apoptosis-related proteins was detected by western blot analysis. ${ }^{*} \mathrm{P}<0.05$ vs. the control. ESCC, esophageal squamous cell carcinoma.

arrest in ESCC cells were investigated using NAC. Flow cytometric results revealed that $2 \mathrm{~h}$ pretreatment with $10 \mathrm{mM}$ NAC could reverse neferine-induced apoptosis (Fig. 5A and B), downregulate the expression of apoptotic-related proteins, cleaved caspase-3, cleaved caspase- 9 and cleaved PARP (Fig. 5C and D) and decrease the neferine-induced $\mathrm{G}_{2} / \mathrm{M}$ cell cycle arrest (Fig. 5E and F). These results indicated that neferine may affect apoptosis and the cell cycle of ESCC cells by inducing ROS accumulation.

Neferine triggers cell cycle arrest and apoptosis by activating the JNK pathway. The aforementioned findings suggested that neferine induced activation of the JNK pathway, which is closely associated with cell proliferation, the cell cycle and apoptosis. Consequently, the effects of neferine-mediated activation of the JNK pathway in apoptosis and cycle arrest in ESCC cells were explored, using SP, an inhibitor of the JNK pathway. Flow cytometric results revealed that $2 \mathrm{~h}$ pretreatment with $15 \mu \mathrm{M}$ SP could reverse neferine-induced apoptosis (Fig. 6A and B) and downregulate the expression of apoptotic-related proteins; cleaved caspase-3, cleaved caspase-9 and cleaved PARP (Fig. 6C and D). In addition, SP-mediated JNK inhibition resulted in a decrease of $\mathrm{G}_{2} / \mathrm{M}$ cell cycle arrest (Fig. 6E and F). These results indicated that neferine may affect apoptosis and the cell cycle of ESCC cells by regulating activation of the JNK signaling pathway.

Neferine triggers the ROS-mediated JNK pathway with Nrf2 inhibition. It was further explored whether ROS participates in the activation of the JNK signaling pathway. Results of the western blot assay revealed that NAC successfully reversed the phosphorylation levels of JNK induced by neferine (Fig. 7A and B). However, SP failed to reduce neferine-induced ROS production (Fig. 7C). These results indicated that neferine induced activation of the JNK signaling pathway by stimulating production of ROS. In addition, it was observed that neferine 
A

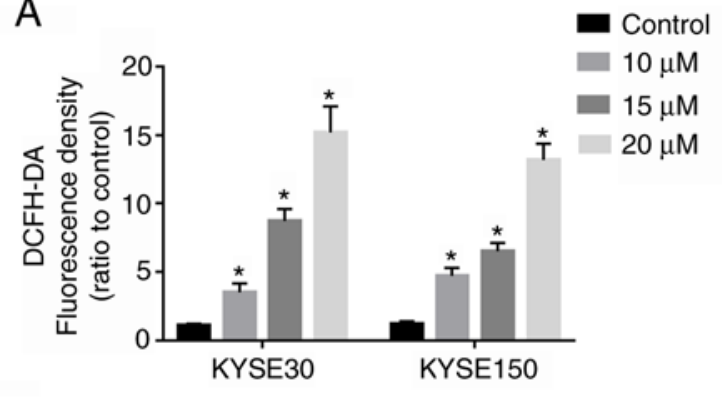

$\mathrm{B}$

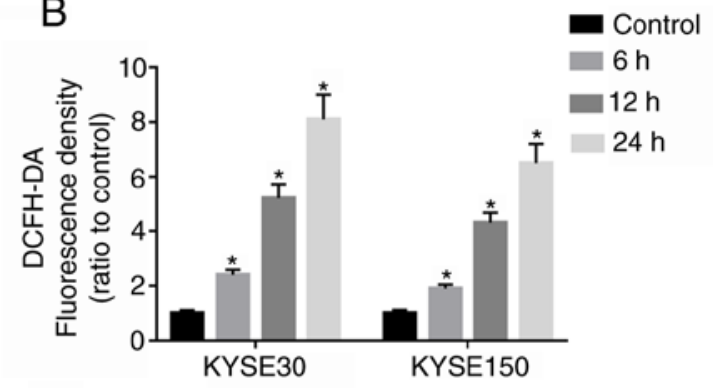

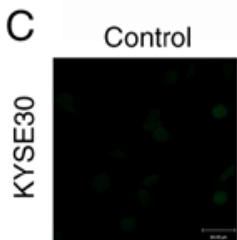
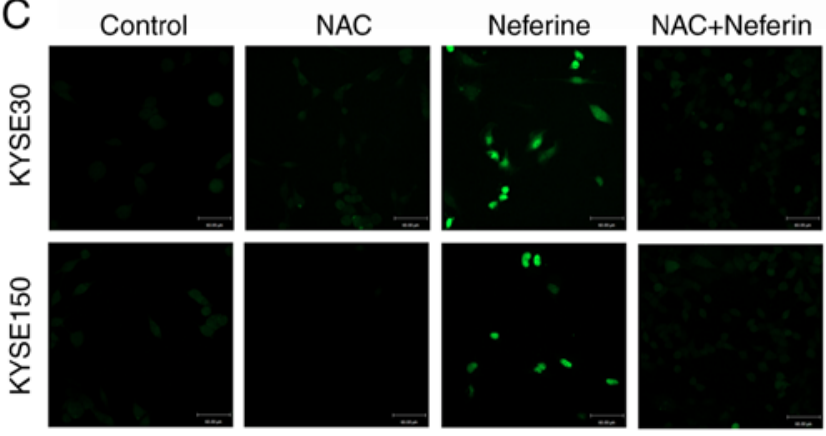
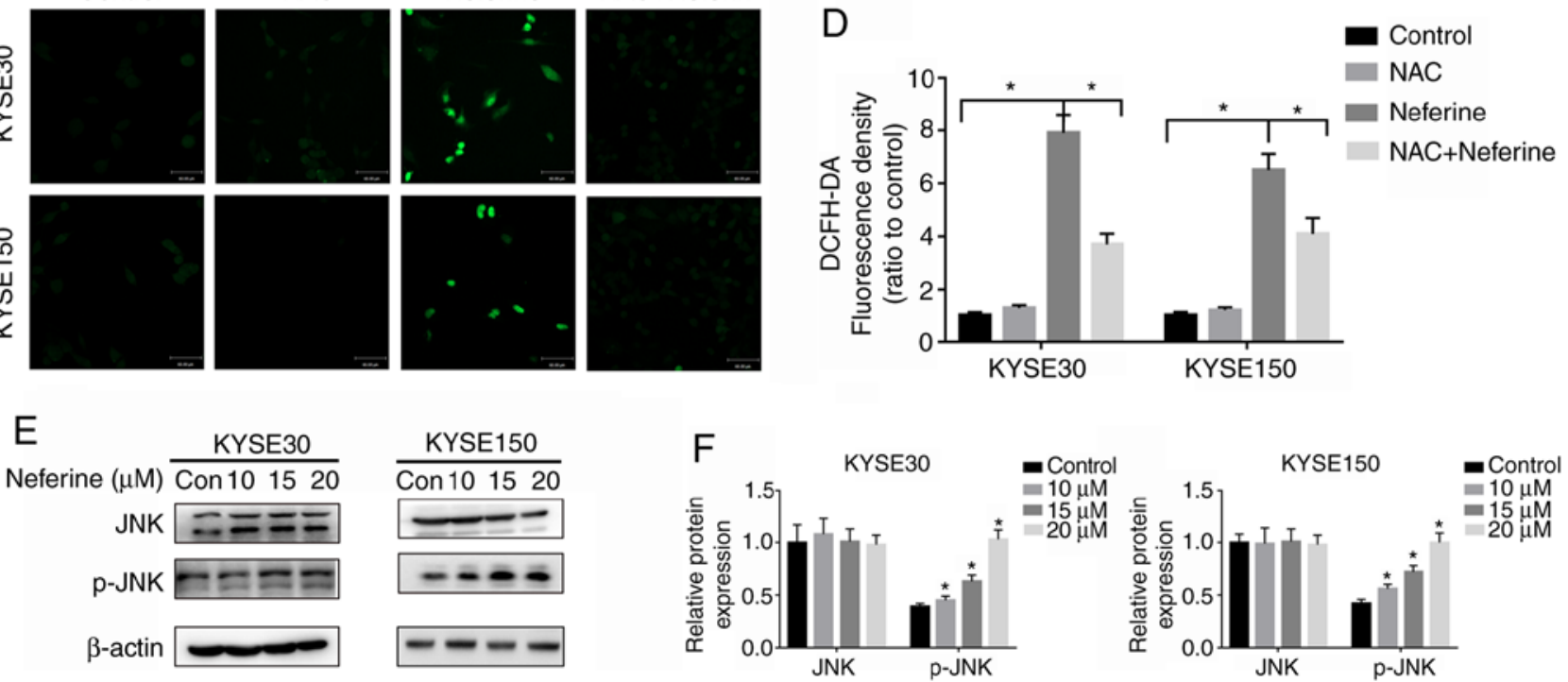

Figure 4. Neferine induces the production of ROS and activates the JNK pathway in ESCC cells. KYSE30 and KYSE150 cells were pretreated with (A) 10 , 15 and $20 \mu \mathrm{M}$ neferine for $12 \mathrm{~h}$ and incubated with (B) $15 \mu \mathrm{M}$ for 6, 12 and $24 \mathrm{~h}$. The ROS levels were assessed using a flow cytometer. (C and D) KYSE30 and KYSE150 cells were treated with $15 \mu \mathrm{M}$ neferine with or without $10 \mathrm{mM}$ NAC for $12 \mathrm{~h}$, and the ROS levels were determined using fluorescence microscopy. (E and F) KYSE30 and KYSE150 cells were treated with neferine (0, 10, 15 and $20 \mu \mathrm{M}$ ) for $24 \mathrm{~h}$, and the expression of JNK and p-JNK was detected by western blotting. ${ }^{*} \mathrm{P}<0.05$ vs. the control or otherwise indicated in the image. ROS, reactive oxygen species; JNK, c-Jun N-terminal kinase; ESCC, esophageal squamous cell carcinoma; NAC, N-acetyl cysteine; p-JNK, phosphorylated JNK.

inhibited the expression of antioxidant, Nrf2 (Fig. 7D and E). Specifically, ESCC cells pretreated with Nrf2 activator, tBHQ, partially offset the proliferation inhibition effect of neferine on ESCC, compared with the control group (Fig. 7F). These data indicated that neferine could inhibit Nrf2 expression, stimulate production of ROS and activate the JNK signaling pathway. Moreover, the compound could also induce apoptosis and cell cycle arrest in ESCC, which indicates a potential role in in management of esophageal carcinoma.

\section{Discussion}

Esophageal squamous cell carcinoma is an aggressive malignancy (1). Currently, ESCC patients are mainly treated using surgical approaches, as well as by radiotherapy or chemotherapy, depending on the pathological results. Although chemotherapeutic drugs improve outcomes of esophageal carcinoma patients to a certain extent, the prognosis of ESCC remains dismal (2). Although several antitumor drugs such as paclitaxel (6), vincristine (8) and colchicine (31), are available for clinical application, they have limited efficacy in killing tumor cells. This indicates that more effective anticancer drugs should be designed to satisfy this unmet clinical need. In the present study, the antitumor effect of neferine, an alkaloid extracted from lotus leaves on ESCC was investigated. The results revealed that it inhibited proliferation of esophageal carcinoma cells, thereby causing $\mathrm{G}_{2} / \mathrm{M}$ phase arrest and inducing apoptosis. In addition, neferine activated the JNK signaling pathway by stimulating production of a high level of ROS and inhibiting Nrf2. Overall, these findings indicated that neferine possesses a significant anti-esophageal carcinoma effect.

Targeting the cell cycle has been a major approach in the development of cancer treatments. Consequently, numerous cell cycle-specific agents, including methotrexate, vinblastine and cytarabine $(32,33)$, have been identified and are widely applied in the clinical management of malignant tumors. Previous studies have revealed that neferine inhibits progression of multiple malignancies, through cell cycle arrest, although this effect varies (12). For instance, Pham et al revealed that neferine hindered proliferation of human neuroblastoma by blocking $\mathrm{G}_{2} / \mathrm{M}$ cell cycle progression (9), while $\mathrm{Xu}$ et al revealed that it could cause G1-phase arrest in ovarian carcinoma cells by upregulating p21 and p27 (11). In the present study, it was observed that neferine treatment increased the $\mathrm{G}_{2} / \mathrm{M}$ phase in ESCC cells by downregulating the expression of cyclin B1 

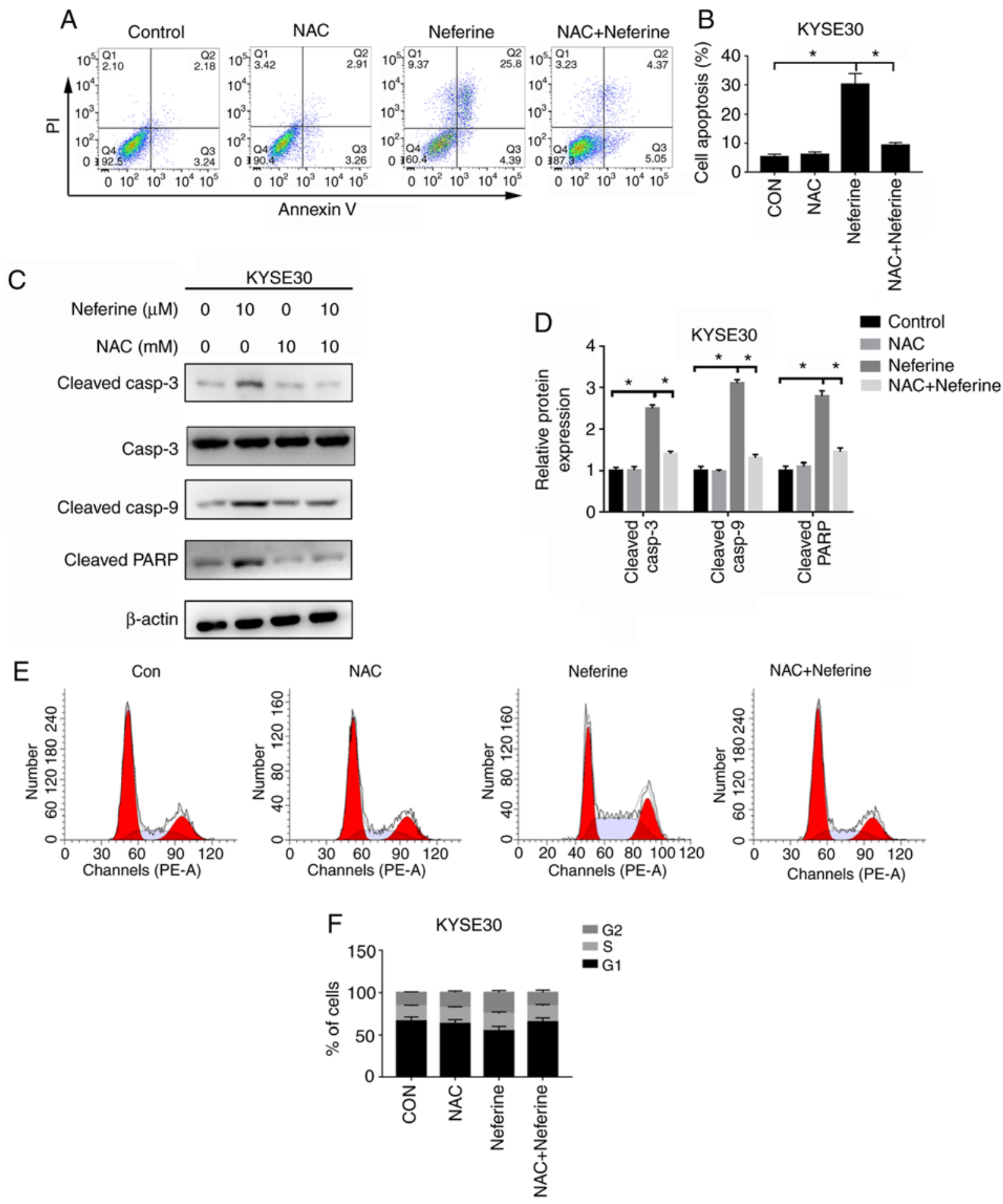

Figure 5. Effect of the JNK pathway in neferine-induced apoptosis and cell cycle arrest. KYSE30 were treated with $15 \mu \mathrm{M}$ neferine with or without $10 \mathrm{mM}$ NAC for $12 \mathrm{~h}$, and (A and B) cell apoptosis was determined by flow cytometry. (C and D) The expression levels of apoptosis-related proteins were determined by western blot analysis. (E and F) The distribution of the cell cycle as determined by flow cytometry. ${ }^{\text {}} \mathrm{P}<0.05$ vs. the control or otherwise indicated in the image. JNK, c-Jun N-terminal kinase; NAC, N-acetyl cysteine.

while upregulating that of $\mathrm{p} 21$. The $\mathrm{Cdk} 1 /$ cyclin $\mathrm{B} 1$ complex is a crucial initiator of mitosis, and has been reported to coordinate $\mathrm{G}_{2} / \mathrm{M}$ progression (34). Studies have revealed that activation of this complex can be blocked by upregulating p21 $(34,35)$. In the present study, neferine suppressed proliferation of tumor cells, by blocking cell cycle progression, although the mechanisms underlying this phenomenon are unclear and require further investigation.
Apoptosis, also known as programmed cell death, was proposed by Kerr et al and refers to an active and inherent programmed phenomenon (36). During this process, cells maintain a balance of quantity through proliferation and apoptosis, but once this balance is broken, it results in certain diseases, such as cancer. Understanding the relationship between apoptosis and cancer development presents new insights into development of novel strategies for treatment 
A
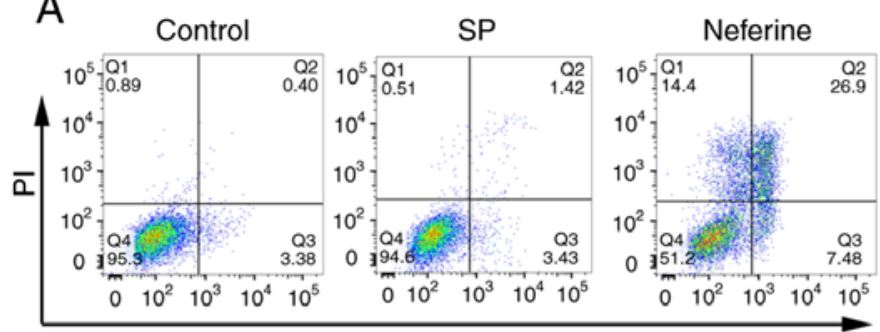

Annexin V
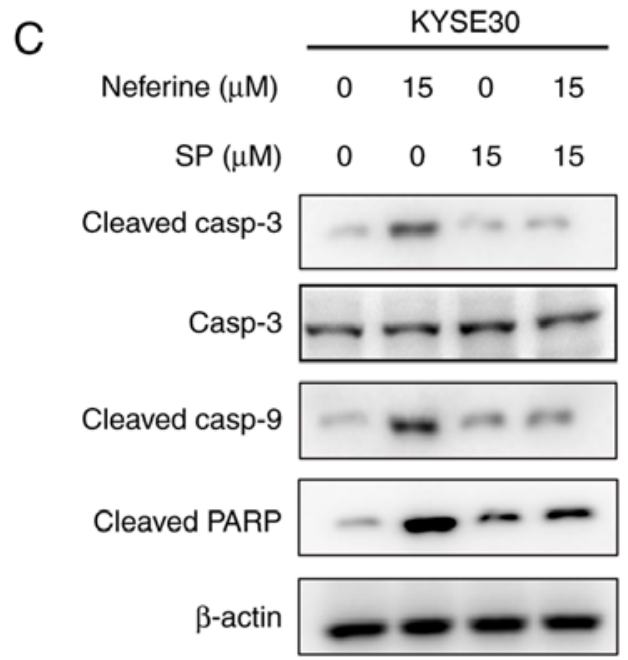

E
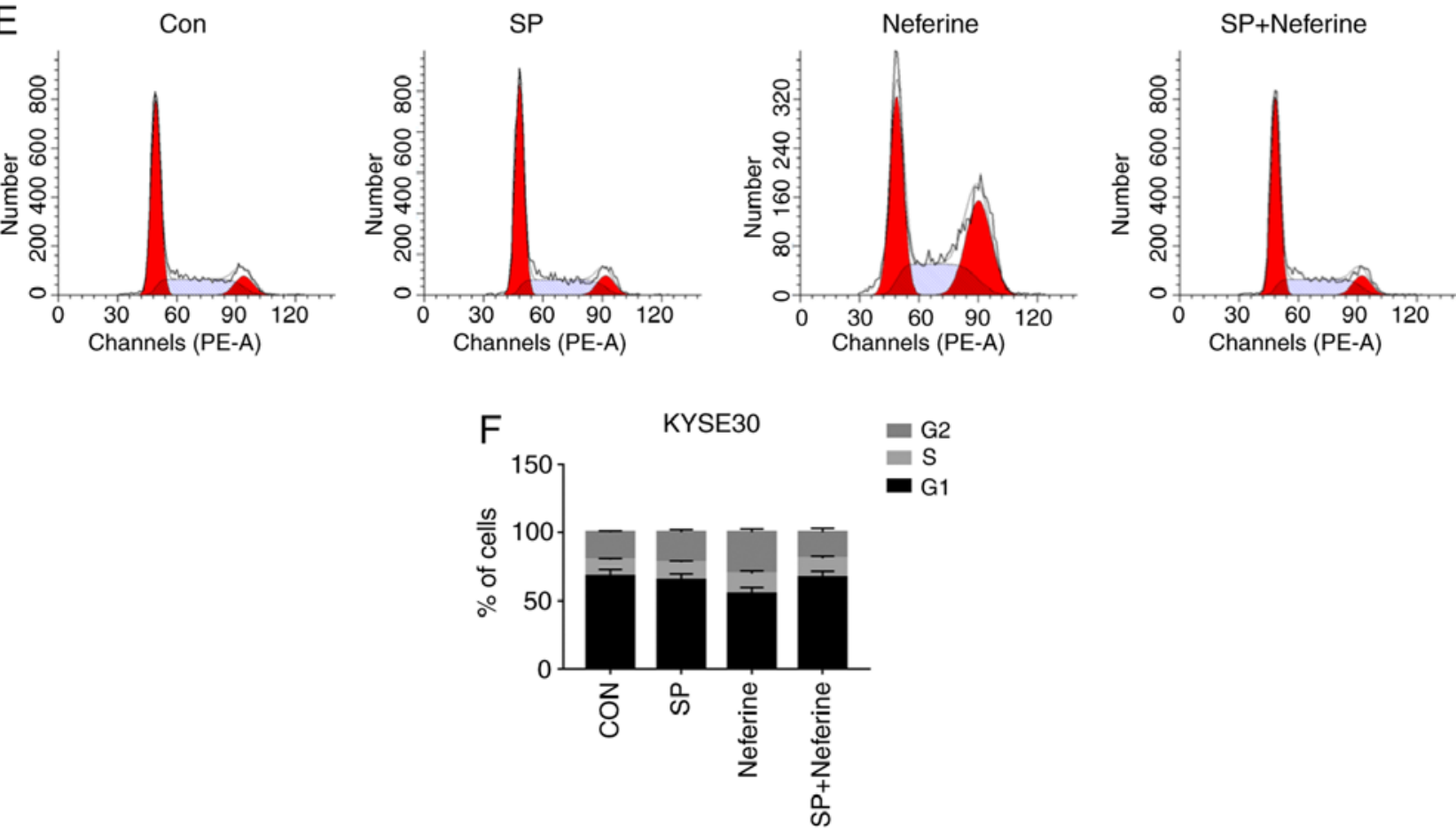

Figure 6. Role of ROS in neferine-induced apoptosis and cell cycle arrest. KYSE30 cells were treated with $15 \mu \mathrm{M}$ neferine with or without $10 \mathrm{mM}$ NAC for $12 \mathrm{~h}$, and then (A and B) cells apoptosis was determined by flow cytometry. (C and D) The expression levels of apoptosis-related proteins by western blot analysis. (E and F) Determination of the cell cycle distribution by flow cytometry. "P<0.05 vs. the control or otherwise indicated in the image. ROS, reactive oxygen species.

of the disease. Consequently, a variety of anticancer drugs have recently been revealed to inhibit progression of tumor cells by inducing apoptosis. For instance, schisantherin (37) hindered proliferation of human gastric cancer cells by
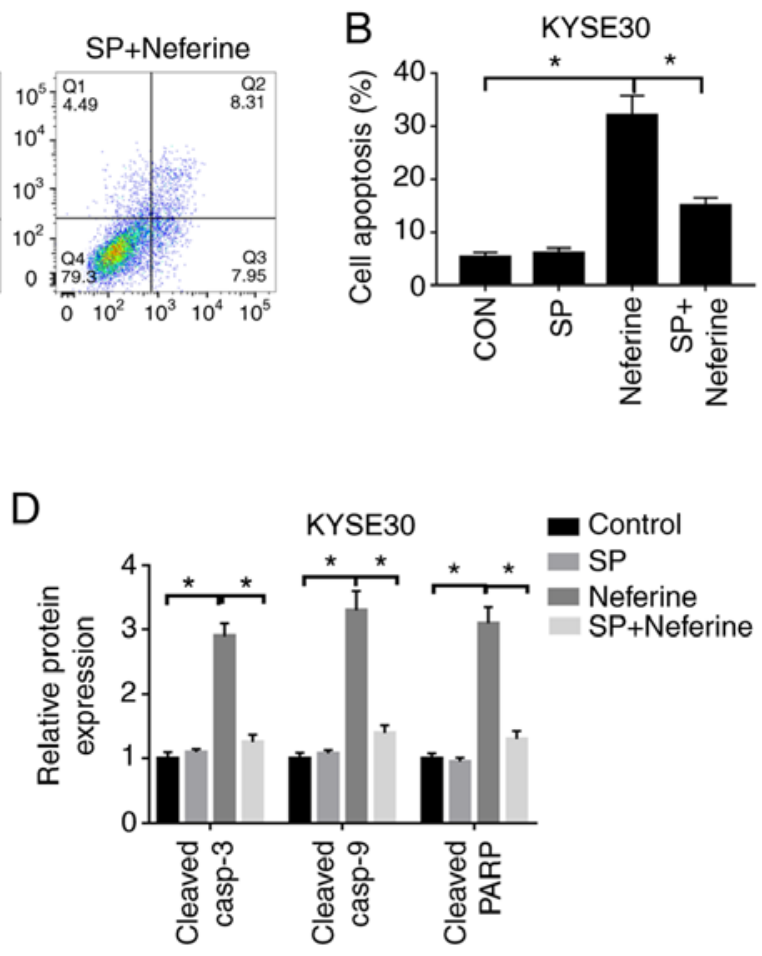
A

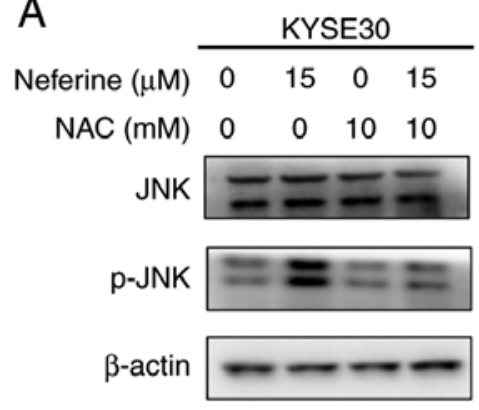

D

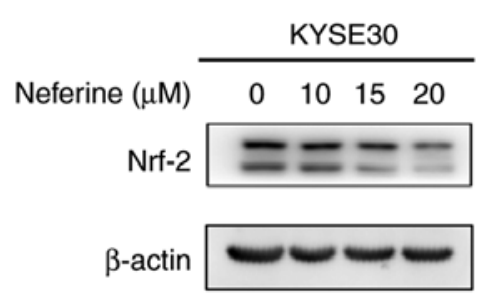

B

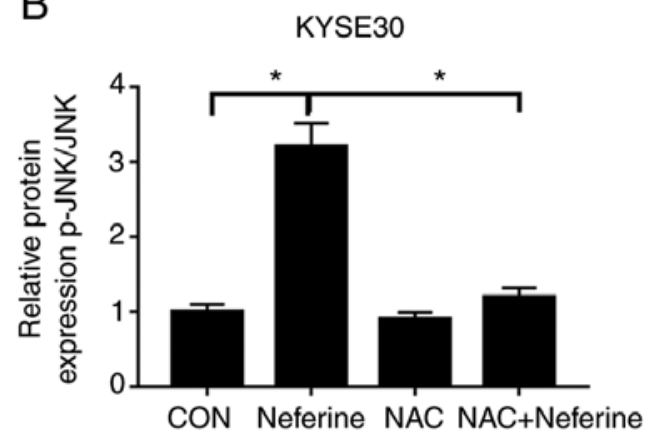

E

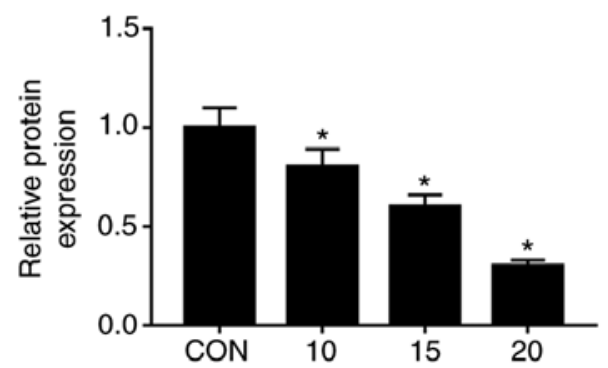

C

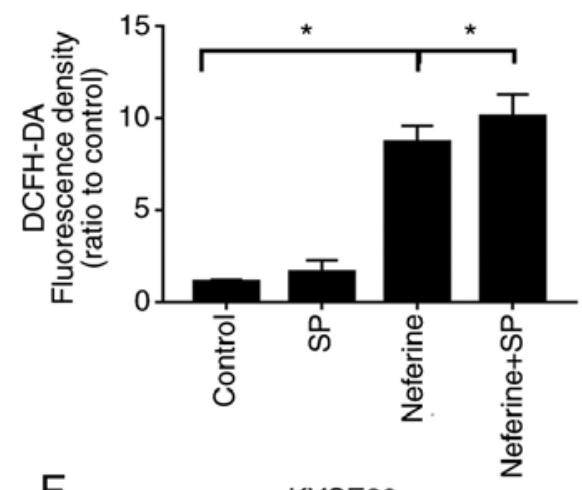

F

KYSE30

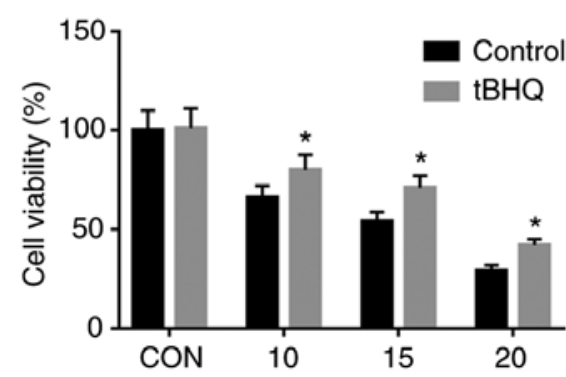

Figure 7. Neferine induces ROS production and activates JNK signaling by inhibiting Nrf2 expression. (A and B) Cells were treated with $15 \mu \mathrm{M}$ neferine with or without $10 \mathrm{mM} \mathrm{NAC}$ for $12 \mathrm{~h}$, and the expression of p-JNK and JNK was determined by western blotting. (C) KYSE30 cells were treated with $15 \mu \mathrm{M}$ neferine with or without $15 \mathrm{mM} \mathrm{SP}$ for $12 \mathrm{~h}$, and the ROS levels were assessed using flow cytometry. (D and E) Cells were treated with 10,15 and $20 \mu \mathrm{M}$ neferine for $12 \mathrm{~h}$, and the expression of Nrf2 was determined by western blot analysis. (F) Cells were treated with 0,10, 15 and $20 \mu \mathrm{M}$ of neferine, with or without $20 \mu \mathrm{M}$ tBHQ for $12 \mathrm{~h}$, and then cell viability was determined with a CCK-8 assay. "P<0.05 vs. the control or otherwise indicated in the image. ROS, reactive oxygen species; JNK, c-Jun N-terminal kinase; NAC, N-acetyl cysteine; SP, SP600125; tBHQ, tert-butylhydroquinone.

caspase-3/9 and PARP, and also resulted in the downregulation of Bcl-2. Collectively, these results indicated that neferine induced the apoptosis pathway in ESCC.

Recent studies have shown that ROS accelerates the death of tumor cells, thereby effectively treating cancer $(38,39)$. High production of ROS decreases the mitochondrial transmembrane potential and the release of cytochrome $c$, which then activates a series of caspases, thereby inducing cell apoptosis. In the present study, it was observed that neferine triggered ROS production in a time- and dose-dependent manner, whereas pretreatment with NAC, an inhibitor of ROS, reversed neferine-induced cell cycle arrest and apoptosis. In addition to apoptosis, neferine-triggered ROS production resulted in cell cycle arrest.

Stress-activated kinases, JNK, are activated by natural compounds such as longikaurin A (40), isoliensinine (41) and ampelopsin (42). In addition, studies have revealed that ROS activates the JNK signaling pathway and promotes tumor cell apoptosis $(17,38,43)$. Similarly, in the present study it was revealed that neferine treatment increased the levels of JNK phosphorylation in esophageal carcinoma cells. Conversely, pretreatment with SP, an inhibitor of the JNK pathway, partially offset neferine-induced cell cycle arrest and apoptosis. Furthermore, pretreatment with NAC attenuated activation of the JNK pathway, however the addition of the JNK inhibitor failed to reduce increase of nerefine-induced ROS. These results indicated that neferine induced cell cycle arrest and apoptosis through the ROS-mediated JNK pathway.
The present results revealed that neferine-treated esophageal carcinoma cells exhibited downregulation of Nrf2 expression, and this was partially offset by Nrf2 activator tBHQ. It was therefore hypothesized, that neferine may inhibit the expression of Nrf2, induce production of ROS and activate the JNK signaling pathway, thus causing cell cycle arrest and inducing apoptosis. Nrf2 is an antioxidant factor that regulates cellular redox state and is highly expressed in a variety of tumor cells (44). In addition, this factor induces and regulates the expression of a series of antioxidant protein groups, and has been found to reduce cell damage caused by ROS and electrophiles, thereby maintaining the cell in a stable state and the dynamic balance of redox reaction $(27,44,45)$. However, there are certain limitations to the present study. All the experiments were performed at the cellular level and the effect of neferine on esophageal carcinoma requires in vivo validation in a future study.

In summary, the present study revealed that neferine can successfully suppress the growth of ESCC cells by blocking cell cycle progression and triggering apoptosis. Furthermore, ROS production and JNK phosphorylation are required in arresting cell cycle progression and triggering apoptosis. Overall, neferine may be a suitable candidate for developing novel therapies for treatment of ESCC.

\section{Acknowledgements}

Not applicable. 


\section{Funding}

No funding was received.

\section{Availability of data and materials}

The datasets used and/or analyzed during the current study are available from the corresponding author on reasonable request.

\section{Authors' contributions}

KA and YZ designed the study, performed the laboratory experiments and wrote the manuscript. YL was responsible for the statistical analysis. SY, ZH, MC, GuL and CD assisted with the experiments and data analysis. JG and GaL reviewed and edited the manuscript. All authors have read and approved the final manuscript and agree to be accountable and agree to be accountable for all aspects of the research in ensuring that the accuracy or integrity of any part of the work are appropriately investigated and resolved.

\section{Ethics approval and consent to participate}

Not applicable.

\section{Patient consent for publication}

Not applicable.

\section{Competing interests}

The authors declare that they have no competing interests.

\section{References}

1. Zhu X, Li Z,Li T,Long F, Lv Y, Liu L, Liu X and Zhan Q: Osthole inhibits the PI3K/AKT signaling pathway via activation of PTEN and induces cell cycle arrest and apoptosis in esophageal squamous cell carcinoma. Biomed Pharmacother 102: 502-509, 2018

2. Yuan X, Liu Y, Li G, Lan Z, Ma M, Li H, Kong J, Sun J, Hou G, Hou X, et al: Blockade of immune-checkpoint B7-H4 and lysine demethylase 5B in esophageal squamous cell carcinoma confers protective immunity against $P$. gingivalis infection. Cancer Immunol Res 7: 1440-1456, 2019.

3. Yuan H, Zhou W, Yang Y, Xue L, Liu L and Song Y: ISG15 promotes esophageal squamous cell carcinoma tumorigenesis via c-MET/Fyn/ $\beta$-catenin signaling pathway. Exp Cell Res 367: 47-55, 2018.

4. Chen W, Zheng R, Baade PD, Zhang S, Zeng H, Bray F, Jemal A, $\mathrm{Yu}$ XQ and He J: Cancer statistics in China, 2015. CA Cancer J Clin 66: 115-132, 2016.

5. Zhu L and Chen L: Progress in research on paclitaxel and tumor immunotherapy. Cell Mol Biol Lett 24: 40, 2019.

6. Kundranda MN and Niu J: Albumin-bound paclitaxel in solid tumors: Clinical development and future directions. Drug Des Devel Ther 9: 3767-3777, 2015.

7. Scherz A, Feller K, Berezowska S, Genitsch V and Zweifel M: Successful treatment of pituitary germinoma with etoposide cisplatin, vincristine, methotrexate and bleomycin chemotherapy without radiotherapy. Anticancer Res 37: 3111-3115, 2017.

8. Zhu B, Yu L and Yue Q: Co-delivery of vincristine and quercetin by nanocarriers for lymphoma combination chemotherapy. Biomed Pharmacother 91: 287-294, 2017.

9. Pham DC, Chang YC, Lin SR, Fuh YM, Tsai MJ and Weng CF: FAK and S6K1 inhibitor, neferine, dually induces autophagy and apoptosis in human neuroblastoma cells. Molecules 23: 3110 , 2018.
10. Deng G, Zeng S, Ma J, Zhang Y, Qu Y, Han Y, Yin L, Cai C, Guo $\mathrm{C}$ and Shen $\mathrm{H}$ : The anti-tumor activities of Neferine on cell invasion and oxaliplatin sensitivity regulated by EMT via Snail signaling in hepatocellular carcinoma. Sci Rep 7: 41616, 2017.

11. Xu L, Zhang X, Li Y, Lu S, Lu S, Li J, Wang Y, Tian X, Wei JJ, Shao $C$ and Liu Z: Neferine induces autophagy of human ovarian cancer cells via p38 MAPK/JNK activation. Tumour Biol 37: 8721-8729, 2016

12. Zhang X, Liu Z, Xu B, Sun Z, Gong Y and Shao C: Neferine, an alkaloid ingredient in lotus seed embryo, inhibits proliferation of human osteosarcoma cells by promoting p38 MAPK-mediated p21 stabilization. Eur J Pharmacol 677: 47-54, 2012.

13. Cannell IG, Merrick KA, Morandell S, Zhu CQ, Braun CJ, Grant RA, Cameron ER, Tsao MS, Hemann MT and Yaffe MB: A pleiotropic RNA-binding protein controls distinct cell cycle checkpoints to drive resistance of p53-defective tumors to chemotherapy. Cancer Cell 28: 831, 2015.

14. Pan X, Liu W, Chai Y, Hu L, Wang J and Zhang Y: Identification of Hub genes in atypical teratoid/rhabdoid tumor by bioinformatics analyses. J Mol Neurosci 2020 (Online ahead of print).

15. Saldivar JC, Hamperl S, Bocek MJ, Chung M, Bass TE, Cisneros-Soberanis F, Samejima K, Xie L, Paulson JR, Earnshaw WC, et al: An intrinsic S/G2 checkpoint enforced by ATR. Science 361: 806-810, 2018.

16. Foijer F, Wolthuis RM, Doodeman V, Medema RH and te Riele H: Mitogen requirement for cell cycle progression in the absence of pocket protein activity. Cancer Cell 8: 455-466, 2005.

17. Liu W, Chai Y, Hu L, Wang J, Pan X, Yuan H, Zhao Z, Song Y and Zhang Y: Polyphyllin VI induces apoptosis and autophagy via reactive oxygen species mediated JNK and P38 activation in Glioma. Onco Targets Ther 13: 2275-2288, 2020.

18. Carneiro BA and El-Deiry WS: Targeting apoptosis in cancer therapy. Nat Rev Clin Oncol 2020 (Online ahead of print).

19. Al Shahrani M, Balasubramaniam M, Alshahrani MY, Saif A, Dera AA, Alasmari S, Abohassan M, Makkawi M, Radhakrishnan S and Rajagopalan P: Computational and in vitro characterization of ICY-5: A potential candidate promoting mitochondrial apoptosis via the c-MET and STAT3 pathways. J Cell Physiol 2020 (Online ahead of print).

20. Buckley AM, Lynam-Lennon N, O'Neill H and O'Sullivan J: Targeting hallmarks of cancer to enhance radiosensitivity in gastrointestinal cancers. Nat Rev Gastroenterol Hepatol 17: 298-313, 2020.

21. Del Re DP, Amgalan D, Linkermann A, Liu Q and Kitsis RN: Fundamental mechanisms of regulated cell death and implications for heart disease. Physiol Rev 99: 1765-1817, 2019.

22. Soteriou D and Fuchs Y: A matter of life and death: stem cell survival in tissue regeneration and tumour formation. Nat Rev Cancer 18: 187-201, 2018.

23. Fendt SM and Lunt SY: Dynamic ROS regulation by TIGAR: Balancing anti-cancer and pro-metastasis effects. Cancer Cell 37: 141-142, 2020.

24. Zhou Z, Song J, Nie L and Chen X: Reactive oxygen species generating systems meeting challenges of photodynamic cancer therapy. Chem Soc Rev 45: 6597-6626, 2016.

25. Wagner EF and Nebreda AR: Signal integration by JNK and p38 MAPK pathways in cancer development. Nat Rev Cancer 9: 537-549, 2009.

26. Kahroba H, Shirmohamadi M, Hejazi MS and Samadi N: The Role of Nrf2 signaling in cancer stem cells: From stemness and self-renewal to tumorigenesis and chemoresistance. Life Sci 239: 116986, 2019.

27. Ferino A, Rapozzi V and Xodo LE: The ROS-KRAS-Nrf2 axis in the control of the redox homeostasis and the intersection with survival-apoptosis pathways: Implications for photodynamic therapy. J Photochem Photobiol B 202: 111672, 2020.

28. DeNicola GM, Karreth FA, Humpton TJ, Gopinathan A, Wei C, Frese K, Mangal D, Yu KH, Yeo CJ, Calhoun ES, et al: Oncogene-induced Nrf2 transcription promotes ROS detoxification and tumorigenesis. Nature 475: 106-109, 2011.

29. Yuan D, Huang S, Berger E, Liu L, Gross N, Heinzmann F, Ringelhan M, Connor TO, Stadler M, Meister M, et al: Kupffer cell-derived tnf triggers cholangiocellular tumorigenesis through JNK due to chronic mitochondrial dysfunction and ROS. Cancer Cell 31: 771-789.e6, 2017.

30. Owusu-Ansah E, Yavari A, Mandal S and Banerjee U: Distinct mitochondrial retrograde signals control the G1-S cell cycle checkpoint. Nat Genet 40: 356-361, 2008. 
31. Cui YJ, Ma CC, Zhang CM, Tang LQ and Liu ZP: The discovery of novel indazole derivatives as tubulin colchicine site binding agents that displayed potent antitumor activity both in vitro and in vivo. Eur J Med Chem 187: 111968, 2020.

32. Mondal A, Gandhi A, Fimognari C, Atanasov AG and Bishayee A: Alkaloids for cancer prevention and therapy: Current progress and future perspectives. Eur J Pharmacol 858 172472, 2019.

33. Salehi B, Selamoglu ZS Mileski K, Pezzani R, Redaelli M, Cho WC, Kobarfard F, Rajabi S, Martorell M, Kumar P, et al: Liposomal Cytarabine as Cancer Therapy: From chemistry to medicine. Biomolecules 9: 773: 2019.

34. Lin JH, Ting PC, Lee WS, Chiu HW, Chien CA, Liu CH, Sun LY and Yang KT: Palmitic acid methyl ester induces G2/M arrest in human bone marrow-derived mesenchymal stem cells via the p53/p21 pathway. Stem Cells Int 2019: 7606238, 2019.

35. Kreis NN, Louwen F and Yuan J: Less understood issues: p21(Cip1) in mitosis and its therapeutic potential. Oncogene 34: $1758-1767,2015$.

36. Kerr N, de Rivero Vaccari JP, Dietrich WD and Keane RW: Neural-respiratory inflammasome axis in traumatic brain injury. Exp Neurol 323: 113080, 2020.

37. Wang Z, Yu K, Hu Y, Su F, Gao Z, Hu T, Yang Y, Cao X and Qian F: Schisantherin A induces cell apoptosis through ROS/JNK signaling pathway in human gastric cancer cells. Biochem Pharmacol 173: 113673, 2020.

38. Yuan YL, Jiang N, Li ZY, Song ZZ, Yang ZH, Xue WH, Zhang XJ and Du Y: Polyphyllin VI induces apoptosis and autophagy in human osteosarcoma cells by modulation of ROS/JNK activation. Drug Des Devel Ther 13: 3091-3103, 2019.

39. Zou P, Zhang J, Xia Y, Kanchana K, Guo G, Chen W, Huang Y, Wang Z, Yang S and Liang G: ROS generation mediates the anti-cancer effects of WZ35 via activating JNK and ER stress apoptotic pathways in gastric cancer. Oncotarget 6: 5860-5876, 2015.
40. Che Y, Wang J, Yuan Z, Li Y, Lu Z, Zhang Z, Zhang J, Wan J, Sun H, Chen Z, et al: The therapeutic effects of Longikaurin A, a natural ent-kauranoid, in esophageal squamous cell carcinoma depend on ROS accumulation and JNK/p38 MAPK activation. Toxicol Lett 280: 106-115, 2017.

41. Zhang X, Wang X, Wu T, Li B, Liu T, Wang R, Liu Q, Liu Z, Gong $\mathrm{Y}$ and Shao C: Isoliensinine induces apoptosis in triple-negative human breast cancer cells through ROS generation and p38 MAPK/JNK activation. Sci Rep 5: 12579, 2015.

42. Guo Z, Guozhang H, Wang H, Li Z and Liu N: Ampelopsin inhibits human glioma through inducing apoptosis and autophagy dependent on ROS generation and JNK pathway. Biomed Pharmacother 116: 108524, 2019.

43. Wang H, Zhang T, Sun W, Wang Z, Zuo D, Zhou Z, Li S, Xu J, Yin F, Hua $Y$ and Cai Z: Erianin induces G2/M-phase arrest, apoptosis, and autophagy via the ROS/JNK signaling pathway in human osteosarcoma cells in vitro and in vivo. Cell Death Dis 7: e2247, 2016.

44. Banerjee N, Wang $H$, Wang $G$ and Khan MF: Enhancing the Nrf2 antioxidant signaling provides protection against trichloroethene-mediated inflammation and autoimmune response. Toxicol Sci 175: 64-74, 2020.

45. Zhou XL, Wu X, Zhu RR, Xu H, Li YY, Xu QR, Liu S, Lai SQ, $\mathrm{Xu} \mathrm{X}$, Wan L, et al: Notch1-Nrf2 signaling crosstalk provides myocardial protection by reducing ROS formation. Biochem Cell Biol 98: 106-111, 2020.

This work is licensed under a Creative Commons Attribution-NonCommercial-NoDerivatives 4.0 International (CC BY-NC-ND 4.0) License. 